\title{
Endoscopic Resection of a Rectal Neuroendocrine Tumor: Hybrid Endoscopic Submucosal Dissection
}

\author{
Marta Gravito-Soares ${ }^{a, c}$ Elisa Gravito-Soares ${ }^{a, c}$ Pedro Amaro ${ }^{a}$ Inês Cunha ${ }^{a}$ \\ João Fraga ${ }^{\mathrm{b}}$ Luís Toméa, $\mathrm{c}$ \\ ${ }^{a}$ Gastroenterology Department, Centro Hospitalar e Universitário de Coimbra, Coimbra, Portugal; \\ bPathology Department, Centro Hospitalar e Universitário de Coimbra, Coimbra, Portugal; \\ 'Faculty of Medicine, University of Coimbra, Coimbra, Portugal
}

Keywords

Hybrid endoscopic submucosal dissection .

Neuroendocrine tumor $\cdot$ Rectum

\section{Resseção endoscópica de um tumor neuroendócrino} do recto: disseção submucosa endoscópica híbrida

\section{Palavras Chave}

Disseção submucosa endoscópica híbrida · Tumor neuroendócrino $\cdot$ Recto

A 67-year-old man was referred to our institution due to a $10-\mathrm{mm}$ yellowish subepithelial lesion in the middle rectum incidentally diagnosed during screening colonoscopy. Conventional biopsies showed a well-differentiated neuroendocrine tumor (NET). Abdominopelvic computed tomography and endoscopic ultrasound showed limited submucosal invasion and no locoregion$\mathrm{al} /$ distant metastasis. It was decided to perform a hybrid endoscopic submucosal dissection (ESD) technique. First, submucosal injection was performed using methylene-blue-stained saline containing 1:100,000 epinephrine with adequate lesion lifting; second, a circumferential incision with a 1-2 $\mathrm{mm}$ free margin (ERBE VIO 300D: Endocut I, effect-1) was made using a ClearCut

\section{KARGER}

E-Mail karger@karger.com www.karger.com/pjg
(C) 2018 Sociedade Portuguesa de Gastrenterologia Published by S. Karger AG, Basel

Karcer

Open access

This article is licensed under the Creative Commons AttributionNonCommercial-NoDerivatives 4.0 International License (CC BYNC-ND) (http://www.karger.com/Services/OpenAccessLicense). Usage and distribution for commercial purposes as well as any distribution of modified material requires written permission. knife 2 mm I-type (Finemedix, South Korea); third, a partial submucosal dissection was done, using the same knife and settings; and fourth, an en bloc resection with an oval $15-\mathrm{mm}$ diathermic snare (Olympus, Spain) was performed (Forced Coag, effect-2 $80 \mathrm{~W}$ ) without complications and resection time of 9 min (Fig. 1a-f). Histopathology showed a 9-mm NET G1 (WHO classification, 0 mitoses/10 HPF, Ki-67: 1.8\%; pT1a AJCC stage 1), limited to the submucosa with free lateral $(1.0 \mathrm{~mm})$ and deep $(0.6 \mathrm{~mm})$ resection margins (Fig. $2 \mathrm{a}-\mathrm{g})$. Considering R0 resection of a $<10-\mathrm{mm}$ rectal NET (R-NET), no followup was scheduled.

Despite the increasing incidence due to the widespread use of screening colonoscopy, R-NET are relatively rare and often well differentiated [1-5]. Endoscopic resection plays a central role in the resection of small well-differentiated R-NET $(<10 \mathrm{~mm})$ and selected cases measuring $10-20 \mathrm{~mm}$, given the low risk of metastasis [2-4]. There is no consensus regarding the best endoscopic resection technique $[1,2,4,5]$, including conventional polypectomy, endoscopic mucosal resection (EMR) or ESD. Conventional polypectomy should be avoided as complete resection is often not achieved $[1,4]$ and EMR shows a suboptimal complete resection rate $(30-70 \%)$ due to frequent submucosal involvement affecting mostly the vertical margin $[1,3,5]$. Incomplete resection requires endoscop- 

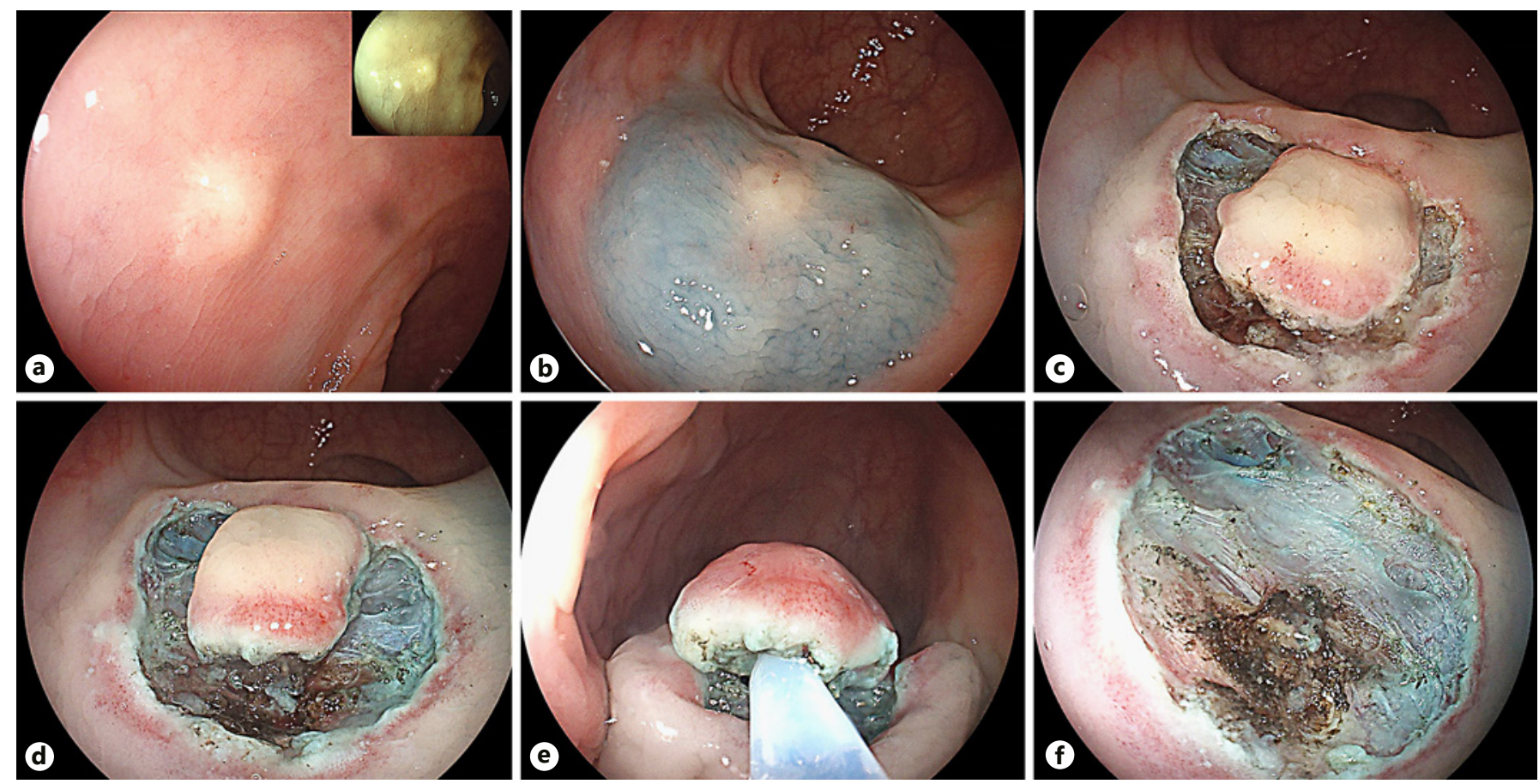

Fig. 1. Hybrid endoscopic submucosal dissection. Endoscopic appearance of a 10-mm yellowish subepithelial lesion in the middle rectum (a, white light and FICE). After submucosal injection of epinephrine-saline mixture $(1: 100,000)$ and methylene blue with adequate lifting of the entire lesion, a circumferential incision was performed using ClearCut knife $2 \mathrm{~mm}$ I-type $(\mathbf{b}, \mathbf{c})$. Partial dissection with ClearCut knife $2 \mathrm{~mm}$ I-type was performed (d). The resection was complemented by en bloc resection using an oval 15-mm diathermic snare (e) with eschar inspection and no complications (f).

ic retreatment with additional difficulty due to submucosal fibrosis, surgery and/or long-term follow-up. Alternatively, ESD has a higher complete resection rate $(80$ $100 \%$ ) with the disadvantage of being a complex and time-consuming procedure and an increased risk of complications [1-4].

Recently, modified EMR (cap- or band ligation-assisted), hybrid EMR/ESD (H-EMR/ESD) techniques, and full-thickness resection [1,3-5] have been proposed to improve in-depth resection of conventional EMR and decrease ESD drawbacks [3-5]. These techniques have a comparable complete resection rate (70-100\%) and similar safety profile as ESD, but with a shorter procedure time [1-4]. H-EMR/ESD has an additional advantage for difficult lesions and tumor size close to $10 \mathrm{~mm}$ or larger, reducing recurrence rate related with modified-EMR due to the limited tumor size that can be aspirated $[2,5]$. However, there are no randomized trials comparing these different resection techniques.

Although ESD, modified EMR, and H-EMR/ESD could be adequate in this case, considering the pericenti- metric endoscopic size of the lesion, the authors opted to perform an H-EMR/ESD in order to secure horizontal free margin. Partial submucosal dissection after complete circumferential incision was additionally performed to provide a deeper submucosal resection and more precise snaring beneath the lesion, theoretically increasing the vertical tumor-free margin without the risk of a full dissection. H-EMR/ESD may be a good treatment choice for small R-NET limited to submucosa, without significantly compromising the completeness, timing, and safety of the procedure.

\section{Statement of Ethics}

Protection of human and animal subjects: The authors declare that no experiments were performed on humans or animals for this study. Confidentiality of data: The authors declare that they have followed the protocols of their work center on the publication of patient data. Right to privacy: The authors declare that no patient data appear in this article. Informed patient consent for publication: Informed consent was obtained for this case report. 

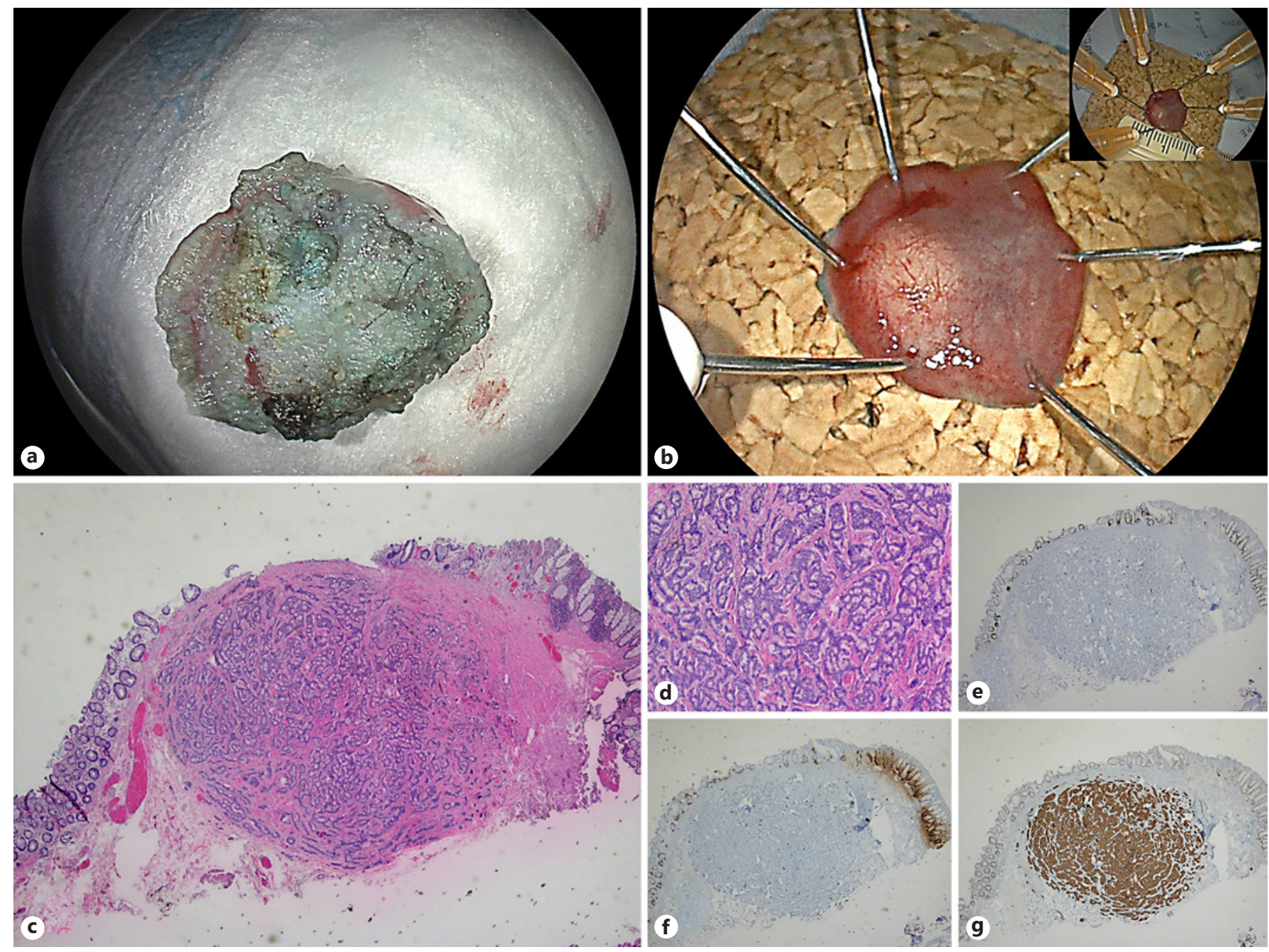

Fig. 2. Appearance of resected specimen (a, b). Pathology of resected specimen showed a neuroendocrine tumor G1 with $9 \mathrm{~mm}$ in diameter, limited to submucosa with free lateral and deep margins (0 mitoses/10 HPF, Ki-67: $1.68 \%$ ). H\&E 20×, 40× (c, d). Ki-67 20× (e). Chromogranin A, 20× (f). Synaptophysin, 20× (g).

\section{Disclosure Statement}

The authors declare no disclosures to report.

\section{Author Contributions}

Marta Gravito-Soares and Elisa Gravito-Soares contributed equally, writing the manuscript and reviewing the literature. Marta Gravito-Soares is the article guarantor. João Fraga reviewed histologic findings. Pedro Amaro, Inês Cunha, and Luís Tomé contributed to critical revision of the manuscript for important intellectual content.

\section{References}

1 Chablaney S, Zator ZA, Kumta NA: Diagnosis and management of rectal neuroendocrine tumors. Clin Endosc 2017;50:530-536.

2 He L, Deng T, Luo H: Efficacy and safety of endoscopic resection therapies for rectal carcinoid tumors: a meta-analysis. Yonsei Med J 2015;56: 72-81.

3 Park SB, Kim HW, Kang DH, et al: Advantage of endoscopic mucosal resection with a cap for rectal neuroendocrine tumors. World J Gastroenterol 2015;21:9387-9393.

4 Bang BW, Park JS, Kim HK, et al: Endoscopic resection for small rectal neuroendocrine tumors: comparison of endoscopic submucosal resection with band ligation and endoscopic submucosal dissection. Gastroenterol Res Pract 2016;2016:6198927.

5 Zhang J, Liu M, Li H, et al: Comparison of endoscopic therapies for rectal carcinoid tumors: endoscopic mucosal resection with circumferential incision versus endoscopic submucosal dissection. Clin Res Hepatol Gastroenterol 2018;42:24-30. 